\title{
Nicotinamide N-Methyltransferase Suppression Participates in Nickel-Induced Histone H3 Lysine9 Dimethylation in BEAS- 2B Cells
}

\author{
Qian Lia Min-Di He ${ }^{a}$ Lin Mao ${ }^{a} \quad$ Xue Wanga Yu-Lin Jiang ${ }^{b}$ Min Lia Yong-Hui Lu ${ }^{a}$ \\ Zheng-Ping Yu ${ }^{a}$ Zhou Zhou ${ }^{a, c}$ \\ aDepartment of Occupational Health, bMolecular Biology Center, State Key Laboratory of Trauma, \\ Burn and Combined Injury, Research Institute of Surgery and Daping Hospital, Third Military Medical \\ University, Chongqing, 'Department of Occupational and Environmental Health, School of Medicine, \\ Guangxi University, Nanning, China
}

\section{Key Words}

Nickel $\bullet \mathrm{NNMT} \cdot$ Histone methylation $\cdot \mathrm{SAM} \cdot \mathrm{NAD}^{+}$

\begin{abstract}
Background: Nickel compounds are well-established human carcinogens with weak mutagenic activity. Histone methylation has been proposed to play an important role in nickel-induced carcinogenesis. Nicotinamide N-methyltransferase (NNMT) decreases histone methylation in several cancer cells by altering the cellular ratio of $\mathrm{S}$-adenosylmethionine (SAM) to S-adenosylhomocysteine (SAH). However, the role of NNMT in nickel-induced histone methylation remains unclear. Methods: BEAS-2B cells were exposed to different concentrations of nickel chloride $\left(\mathrm{NiCl}_{2}\right)$ for $72 \mathrm{~h}$ or $200 \mu \mathrm{M} \mathrm{NiCl}$ for different time periods. Histone $\mathrm{H} 3$ on lysine 9 (H3K9) mono-, di-, and trimethylation and NNMT protein levels were measured by western blot analysis. Expressions of NNMT mRNA and the H3k9me2-associated genes, mitogen-activated protein kinase 3 (MAP2K3) and dickkopf1 (DKK1), were determined by $\mathrm{qPCR}$ analysis. The cellular ratio of nicotinamide adenine dinucleotide $\left(N A D^{+}\right)$to reduced NAD (NADH) and SAM/SAH ratio were determined. Results: Exposure of BEAS-2B cells to nickel increased H3K9 dimethylation (H3K9me2), suppressed the expressions of H3K9me2associated genes (MAP2K3 and DKK1), and induced NNMT repression at both the protein and mRNA levels. Furthermore, over-expression of NNMT inhibited nickel-induced H3K9me2 and altered the cellular SAM/SAH ratio. Additionally, the NADH oxidant phenazine methosulfate (PMS) not only reversed the nickel-induced reduction in NAD $/ \mathrm{NADH}$ but also inhibited the increase in $\mathrm{H} 3 \mathrm{~K} 9 \mathrm{me} 2$. Conclusions: These findings indicate that the repression of NNMT may underlie nickel-induced H3K9 dimethylation by altering the cellular SAM/SAH ratio.

Q. Li and M.-D. He contributed equally to this work.




\section{Cellular Physiology Cell Physiol Biochem 2017;41:2016-2026

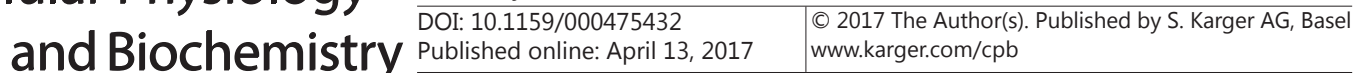 \\ Li et al.: Nickel Induces H3K9me2 by Inhibiting NNMT}

\section{Introduction}

Nickel compounds are classified as lung carcinogens in humans by the International Agency for Research on Cancer (IARC) based on epidemiological and experimental studies [1]. A recent retrospective cohort study reported increased mortality due to lung cancer among nickel refinery workers [2]. In addition, exposure to nickel compounds induced tumors in experimental animals [3] and transformed mammalian cells in vitro [4]. Since numerous conventional mutagenesis assays have found nickel compounds to be weakly mutagenic [5, 6], an epigenetic mechanism has been hypothesized to explain nickel-induced carcinogenesis [7]. Epigenetic changes have been suggested to play a pivotal role in tumor formation and progression [8]. Histone methylation, one of the best-studied epigenetic alterations, can regulate gene expression depending on the post-translational modifications at different residues [9]. Among histone methylations, methylation of lysine 9 in histone H3 (H3K9) has been extensively studied [10]. Repressed promoters are marked by H3K9 dimethylation (H3K9me2), and this critical marker has been linked to transcriptional repression in different cell lines $[11,12]$. The increase in $\mathrm{H} 3 \mathrm{~K} 9 \mathrm{me} 2$ at the gene promoter repressed the expression of the tumor suppressor sprouty homolog2 (SPRY2) and led to nickel-induced anchorage-independent growth and carcinogenesis [13]. Therefore, further clarification of nickel-induced histone methylation will contribute to understanding nickel carcinogenesis.

Nicotinamide N-methyltransferase (NNMT) methylates nicotinamide (NAM) to $N^{1}$ methylnicotinamide (MNAM) using S-adenosylmethionine (SAM) as the methyl group donor and generates S-adenosyl homocysteine (SAH) $[14,15]$. NNMT expression has been shown to correlate with the migration, invasion, proliferation and survival of cancer cells [16-18]. Over-expression of NNMT in cancer cells resulted in hypomethylated histones by altering the cellular SAM/SAH ratio [19]. However, to the best of our knowledge, the effects of nickel on NNMT and the possible role of NNMT in nickel-induced histone methylation are not clear. Nicotinamide adenine dinucleotide (NAD) acts as a coenzyme in metabolic redox reactions. The coenzyme is found in two forms: an oxidized form and reduced form, referred to as $\mathrm{NAD}^{+}$and $\mathrm{NADH}$, respectively [20]. A previous study indicated that nickel exposure could decrease the $\mathrm{NAD}^{+} / \mathrm{NADH}$ ratio in the cortical cortex of mice [21]. Recent studies have shown that NNMT could modulate the cellular SAM/SAH and NAD ${ }^{+}$NADH ratios and subsequently affect histone acetylation and histone methylation $[22,23]$. Taken together, NNMT may play a pivotal role in histone modifications.

Thus, the purpose of this study was to determine whether NNMT participates in nickelinduced histone methylation. We found that nickel-induced NNMT repression was involved in the increase of $\mathrm{H} 3 \mathrm{~K} 9 \mathrm{me} 2$ in BEAS-2B cells. Reversing NNMT suppression could abolish nickel-induced $\mathrm{H} 3 \mathrm{~K} 9 \mathrm{me} 2$ by altering the SAM/SAH ratio. These results illustrate a new molecular mechanism of nickel-induced histone methylation.

\section{Materials and Methods}

\section{Cell culture and viability determination}

Human bronchial epithelial cells (BEAS-2B) were cultured in RPMI 1640 medium (HyClone, Logan, UT, USA) supplemented with $10 \%$ fetal bovine serum (PAN-Biotech), $100 \mathrm{U} / \mathrm{mL}$ penicillin and $100 \mu \mathrm{g} / \mathrm{ml}$ streptomycin (Beyotime, Haimen, Jiangsu, China) at $37^{\circ} \mathrm{C}$ in a $5 \% \mathrm{CO}_{2}$ atmosphere. For dose-dependent and time- dependent experiments of nickel exposure, $\mathrm{NiCl}_{2}$ (Sigma-Aldrich, St. Louis, MO, USA) was added to the media at concentrations of 0 (control), 25, 50, 100, and $200 \mu \mathrm{M}$ for $72 \mathrm{~h}$ or $200 \mu \mathrm{M}$ for $0 \mathrm{~h}, 12 \mathrm{~h}, 24 \mathrm{~h}, 48 \mathrm{~h}$, and $72 \mathrm{~h}$.

Cell viability was measured using the Cell Counting Kit-8 (CCK-8, Dojindo Molecular Technologies, Kumamoto, Japan) according to the manufacturer's instructions. After the treatment, the medium in the 96-well plate was replaced with fresh medium containing $10 \% \mathrm{v} / \mathrm{v}$ CCK-8 solution and further cultured at $37^{\circ} \mathrm{C}$ for $2 \mathrm{~h}$. Following incubation, the absorbance was read at $450 \mathrm{~nm}$ using a microplate reader (Tecan, 


\section{Cellular Physiology Cell Physiol Biochem 2017;41:2016-2026 and Biochemistry Published onlıne: April 13, $2017 \quad \begin{aligned} & \text { DOI: 10.1159/000475432 } 2017 \text { The Author(s). Published by S. Karger AG, Basel } \\ & \text { www.karger.com/cpb }\end{aligned}$}

Li et al.: Nickel Induces H3K9me2 by Inhibiting NNMT

Mannedorf, Switzerland). The results are presented as the percent cell viability assuming that the viability of control cells was $100 \%$.

\section{Western blot}

After the indicated treatments, cells were collected, and protein extracts from whole cells were separated by SDS-PAGE and transferred onto a polyvinylidene fluoridemembrane (PVDF, Bio-Rad, Hercules, CA, USA). The membranes were blocked for $1 \mathrm{~h}$ at room temperature and then incubated overnight at 4 ${ }^{\circ} \mathrm{C}$ with the following primary antibodies: 1:100-diluted NNMT (Santa Cruz Biotechnology, CA, USA) , 1:10,000-diluted H3K9me1, H3K9me2 and H3K9me3 (Abcam, Cambridge, UK), and 1:5,000-diluted $\beta$-actin (Thermo Fisher Scientific, Waltham, MA, USA). The membranes were then incubated with secondary antibodies coupled to horseradish peroxidase for $1 \mathrm{~h}$ at room temperature. The bands were visualized using a chemiluminescence substrate (Millipore, Billerica, MA, USA) and detected using the ChemDoc ${ }^{\mathrm{TM}}$ XRS+ imaging system (Bio-Rad).

\section{Quantitative real-time PCR}

Total RNA was isolated using TRIzol (TaKaRa BioInc., Shiga, Japan). cDNA was synthesized from total RNA by a reverse transcription kit (TaKaRa) according to the manufacturer's protocol. Quantitative real-time PCR was performed according to standard protocols using commercially available primers purchased from Invitrogen (Shanghai, China). GAPDH was utilized as an internal control for mRNA. The primers used in this study were as follows: MAP2K3-forward, 5'-GAGGGAGACGTGTGGATCTG-3' and reverse: 5'-CCGCACGATA GACACAGCAAT-3'; DKK1-forward, 5'-CCTTGAACTCGGTTCTCAATTCC-3' and reverse: 5'-CAATGGTCTGGTACTTATTCCCG-3'; NNMT forward, 5'-ATATTCTGCCTAGACGGTGTGA-3' and reverse: 5'-TCAGTGACGACGATCTCCTTAAA-3'; GAPDH-forward, 5'-TCAAGAAGGTGGTGA AGCAGG-3' and reverse: 5'-AGCGTCAAAGGTGGAGGAGTG-3'. The relative expression levels of each target gene were normalized to the mRNA levels of the GAPDH.

\section{Cell transfection}

The recombinant plasmid pcDNA3.1(+)-NNMT was constructed by Invitrogen. After seeding the cells in 6-well plates and incubation for $24 \mathrm{~h}$, the medium without penicillin/streptomycin was replaced with transfection medium containing $4 \mu \mathrm{g}$ pcDNA3.1(+)-NNMT or pcDNA3.1(+) vector and $8 \mu \mathrm{L}$ Lipofectamine2000 transfection reagent (Invitrogen, Shanghai, China) per well. After $5 \mathrm{~h}$, the transfection medium was replaced with fresh medium without penicillin/streptomycin. The transiently transfected cells were further incubated at $37^{\circ} \mathrm{C}$ for $19 \mathrm{~h}$ and then treated with or without $200 \mu \mathrm{M} \mathrm{NiCl}_{2}$ for another $24 \mathrm{~h}$ and then collected for further experiments.

\section{Biochemical measurements}

The NAD ${ }^{+}$NADH ratio was assessed according to instructions provided by the NAD/NADH Ratio Assay Kit (Promega, USA). Concentrations of SAM and SAH were determined using reversed-phase high performance liquid chromatography (HPLC). The cells $\left(1 \times 10^{7}\right)$ were centrifuged, washed twice with cold PBS, and maintained on ice. The cell pellets were subsequently homogenized in $100 \mu \mathrm{L}$ of $0.4 \mathrm{M}$ ice-cold perchloric acid. Homogenates were centrifuged at $15,000 \mathrm{~g}$ for $15 \mathrm{~min}$ at $4{ }^{\circ} \mathrm{C}$. The supernatants were collected and stored at $-80{ }^{\circ} \mathrm{C}$ until they were analysed. The supernatant of each sample was loaded into a C18 column (SunFire, Ireland) and run by a Waters HPLC system (Milford, MA). The two mobile phases were as follows : Mobile phase A consisted of $50 \mathrm{mM} \mathrm{NaH}_{2} \mathrm{PO}_{4}$ and $8 \mathrm{mM}$ heptanesulfonic acid ( $\left.\mathrm{pH}=3.0\right)$, and Mobile phase B contained methanol. The isocratic elution was achieved at a flow rate of $1 \mathrm{~mL} / \mathrm{min}$ with the following parameters: $80 \%$ Phase A and 20\% Phase B. The total run time was 15 min, and the injection volume was $20 \mu \mathrm{L}$. The absorbance of analytes was recorded by a UV detector at $254 \mathrm{~nm}$. The SAM and SAH standards purchased from Sigma-Aldrich (St. Louis, MO, USA) were used to identify the elution peaks, and the cellular SAM and SAH were calculated by automatic peak area integration.

\section{Statistical analysis}

All data are expressed as mean \pm SEM from at least three independent experiments. GraphPad Prism5 statistical package (GraphPad, SanJose, CA) was used for one-way analysis of variance (ANOVA), and Tukey's test was used for the post hoc comparison of means. Statistical significance was defined when $p<0.05$. 


\section{Results}

Nickel increases H3K9 dimethylation and down-regulates MAP2K3 and DKK1 expressions

To determine the concentration and incubation time of the nickel treatment, BEAS-2B cells were exposed to $\mathrm{NiCl}_{2}$ at 100 and $200 \mu \mathrm{M}$ for $0,12,24,48$ and $72 \mathrm{~h}$ as described in previous studies $[11,13,24,25]$. The CCK-8 assay showed that $200 \mu \mathrm{M} \mathrm{NiCl}_{2}$ did not induce acute toxic effects on cells for up to $72 \mathrm{~h}$ exposure (Fig. 1). To investigate the effects of nickel on histone methylation, we first examined the global H3K9 methylation in BEAS-2B cells after

Fig. 1. Effect of nickel on cell viability in BEAS-2B cells. The cell viability was assessed by the CCK-8 assay. BEAS-2B cells were treated with 100 or $200 \mu \mathrm{M}$ $\mathrm{NiCl}_{2}$ for $0 \mathrm{~h}$ to $72 \mathrm{~h}$. The data are shown as the me$\mathrm{an} \pm \mathrm{SEM}$ of at least three independent experiments.

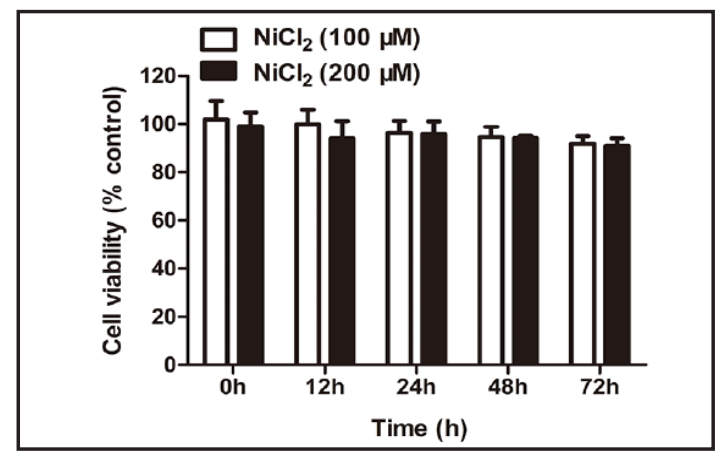

Fig. 2. Nickel exposure induces H3K9 dimethylation and down-regulates $M A P 2 K 3$ and DKK1 expressions. BEAS-2B cells were exposed to various concentrations of $\mathrm{NiCl}_{2}$ for $72 \mathrm{~h}$, and the levels of $\mathrm{H} 3 \mathrm{~K} 9 \mathrm{me} 1$ (A), H3K9me2 (B) and H3K$9 \mathrm{me} 3(\mathrm{C})$ were determined by western blot analysis. (D) Time course change in H3K9me2 following nickel exposure. Nickel exposure time- and dose-dependently down-regulates $M A P 2 K 3$ and $D K K 1$ expressions (E, F). All data are presented as the mean \pm SEM from at least three independent experiments, ${ }^{*} \mathrm{P}<0.05$, compared with the control.

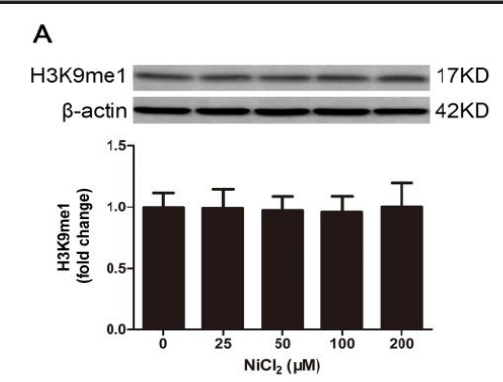

C

B
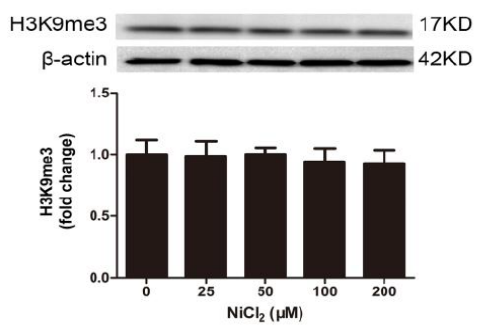

E

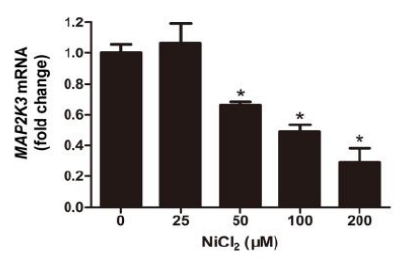

G

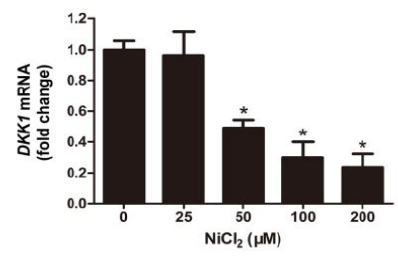

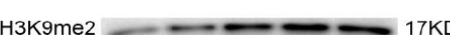
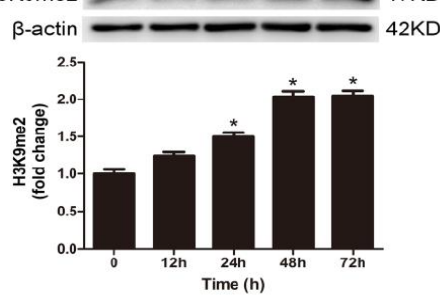

F

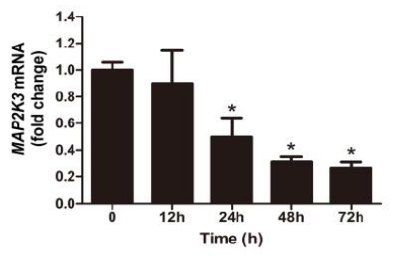

$\mathrm{H}$

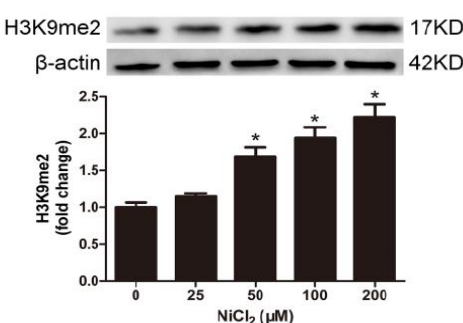

D

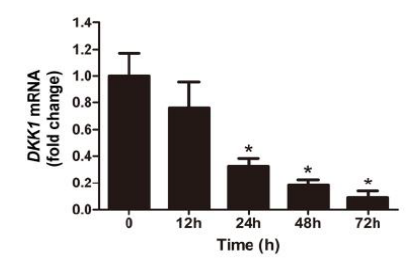


A
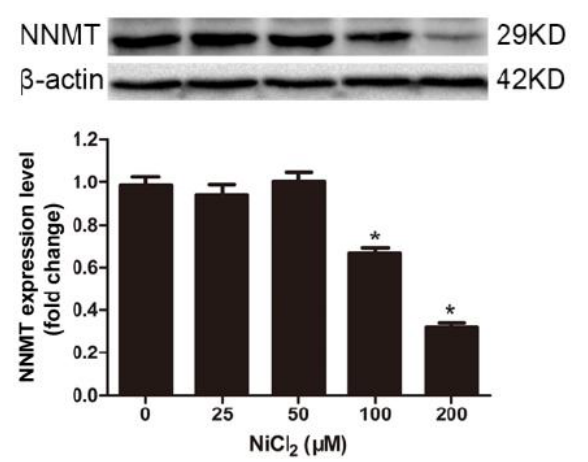

C

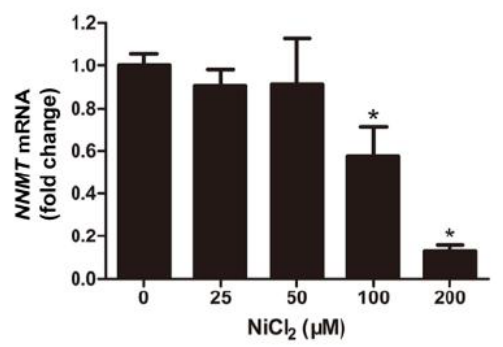

B
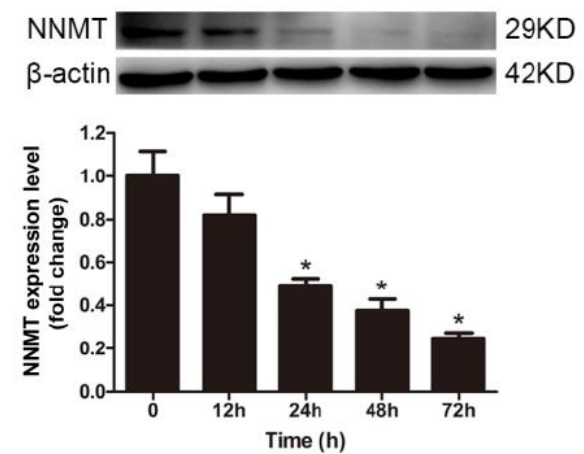

D

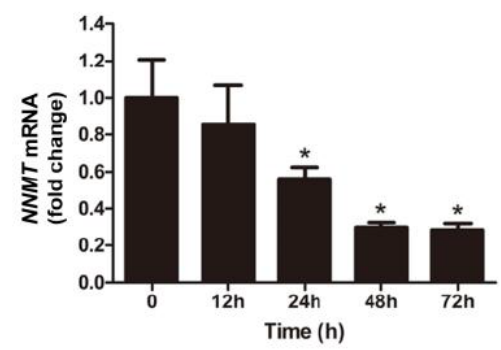

Fig. 3. Nickel exposure suppresses NNMT expression. NNMT protein level determined by western blot (A, B). NNMT mRNA level determined by qRT-PCR (C, D). All data are presented as the mean \pm SEM from at least three independent experiments. ${ }^{*} \mathrm{P}<0.05$, compared with the control.

nickel exposure. Western blot analysis showed that nickel increased H3K9 dimethylation (H3K9me2) but had no effect on H3K9 monomethylation (H3K9me1) and trimethylation (H3K9me3) (Fig. 2A, B and C). The increase in H3K9me2 began at $12 \mathrm{~h}$, peaked at $48 \mathrm{~h}$, and was sustained at $72 \mathrm{~h}$ after nickel exposure (Fig. 2D). To further confirm the changes in H3K9me2, mitogen-activated protein kinase 3 (MAP2K3) and dickkopf1 (DKK1), H3k9me2associated tumor suppressor genes [26-28], were assessed by qRT-PCR. As shown in Fig. $2 \mathrm{E}$ and F, nickel significantly decreased MAP2K3 and DKK1 expressions in dose- and timedependent manners.

\section{Nickel suppresses NNMT expression}

NNMT expression was determined by western blot and qRT-PCR at the protein and gene levels, respectively. Western blot analysis revealed that nickel induced a significant decrease in NNMT expression at the protein level (Fig. 3A and B). Consistent with the protein expression, qRT-PCR results showed that NNMT mRNA was also suppressed in BEAS-2B cells following nickel exposure (Fig. 3C and D).

Over-expression of NNMT abolishes the increase in H3K9me2 induced by nickel

To further confirm that inhibition of NNMT mediates the nickel-induced histone methylation and down-regulation of tumor suppressor genes, an NNMT overexpression plasmid was transfected into BEAS-2B cells. As shown in Fig. 4A to D, NNMT overexpression could reverse nickel-induced $\mathrm{H} 3 \mathrm{~K} 9 \mathrm{me} 2$ and abolish the repression of MAP2K3 and DKK1.

PMS reverses the nickel-induced increase in H3K9me2

To investigate whether $\mathrm{NAD}^{+} / \mathrm{NADH}$ alterations are involved in nickel-induced H3K9 dimethylation in BEAS-2B cells, phenazine methosulfate (PMS), a crucial electron acceptor oxidizing $\mathrm{NADH}$ to $\mathrm{NAD}^{+}$[29], was applied to reverse the decrease in the $\mathrm{NAD}^{+} / \mathrm{NADH}$ ratio 
Fig. 4. Over-expression of NNMT inhibits the nickel-induced increase in H3K9me2 and decrease in $M A P 2 K 3$ and DKK1 expressions. The protein expression of NNMT (A) and H3K9me2 (B) were determined by western blot analysis. mRNA levels of MAP $2 K 3(\mathrm{C})$ and $D K K 1$ (D) were detected by qRT-PCR. All the data are presented as the mean \pm SEM from at least three independent experiments. ${ }^{*} \mathrm{p}<0.05$, compared with the control; $\# \mathrm{p}<0.05$, compared with nickel exposure alone.

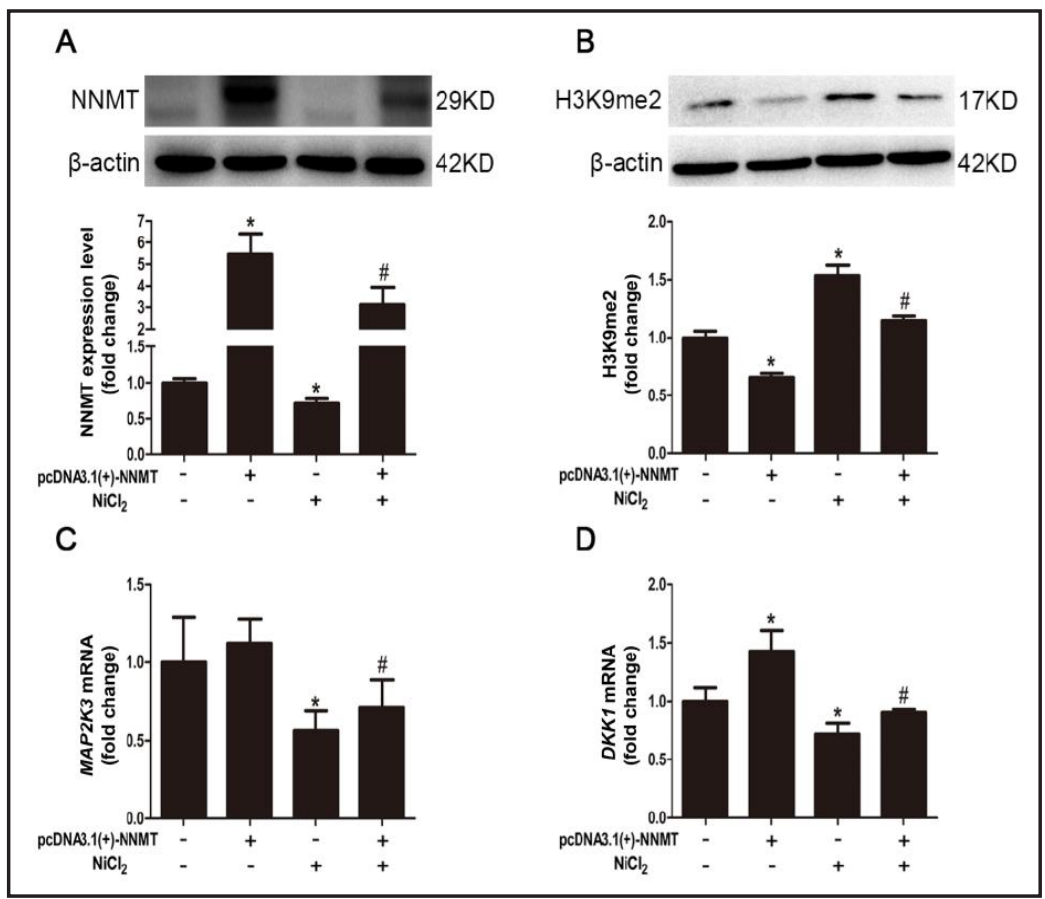

Fig. 5. PMS treatment reverses nickel-induced up-regulation of H3K9me2 and down-regulation of $M A P 2 K 3$ and DKK1 expressions. The $\mathrm{NAD}^{+} / \mathrm{NADH}$ ratio (A) was measured. The H3K9me2 levels (B) was determined by western blot analysis. mRNA levels of MAP2K3 (C), DKK1 (D) were detected by qPCR. All data are presented as the mean \pm SEM from at least three independent experiments. ${ }^{*} \mathrm{p}<0.05$, compared with the control; $\# p<0.05$, compared with nickel exposure alone.
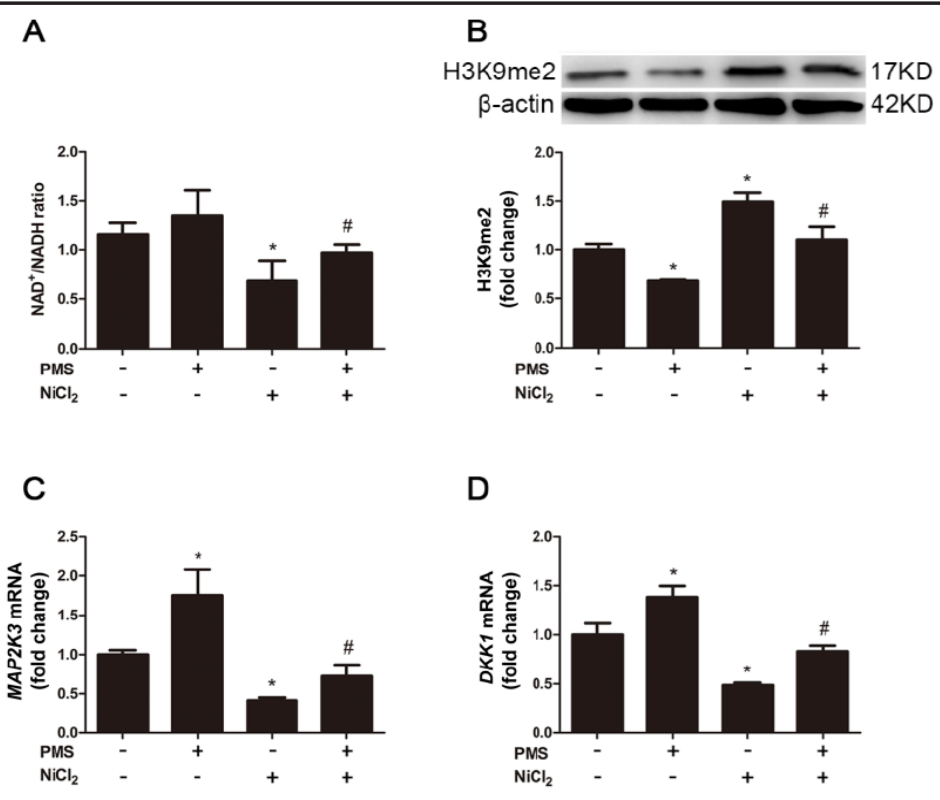

by nickel (Fig. 5A). PMS not only inhibited the increase in H3K9me2 but also abolished the down-regulation of $M A P 2 K 3$ and $D K K 1$ as shown in Fig. 5B, C and D.

NNMT down-regulates $H 3$ K9me2 by altering the SAM/SAH ratio

SAM serves as a universal methyl donor for transmethylation reactions, including histone methylation and is subsequently converted to SAH [30,31]. NNMT has been reported to induce hypomethylated histones in cancer cells by decreasing the ratio of SAM/SAH [19]. To determine whether NNMT regulates nickel-induced H3K9 dimethylation by altering the cellular SAM/SAH ratio in BEAS-2B cells, cellular SAM and SAH levels were determined by HPLC. As shown in Fig. 6A, the retention times for standard SAH and SAM were $4.35 \mathrm{~min}$ and 6.24 min, respectively. NNMT over-expression in BEAS-2B cells resulted in a significant 
Fig. 6. NNMT regulates the SAM/ $\mathrm{SAH}$ ratio in BEAS-2B cells. Chromatograms of a standard mixture of SAM and SAH. The retention times for SAM and SAH were $4.34 \mathrm{~min}$ and 6.25 min, respectively (A). Compared chromatograms of two groups (B and C): pcDNA3.1(+) vector group (black) versus pcDNA3.1(+)-NNMT (blue) and the pcDNA3.1(+) group versus pcDNA3.1(+) $+\mathrm{NiCl}_{2}$ (blue). The SAM/ $\mathrm{SAH}$ ratio is presented as the mean \pmSEM in different groups (D). ${ }^{*} \mathrm{P}<0.05$, compared with the control; $\# \mathrm{p}<0.05$, compared with nickel exposure alone.

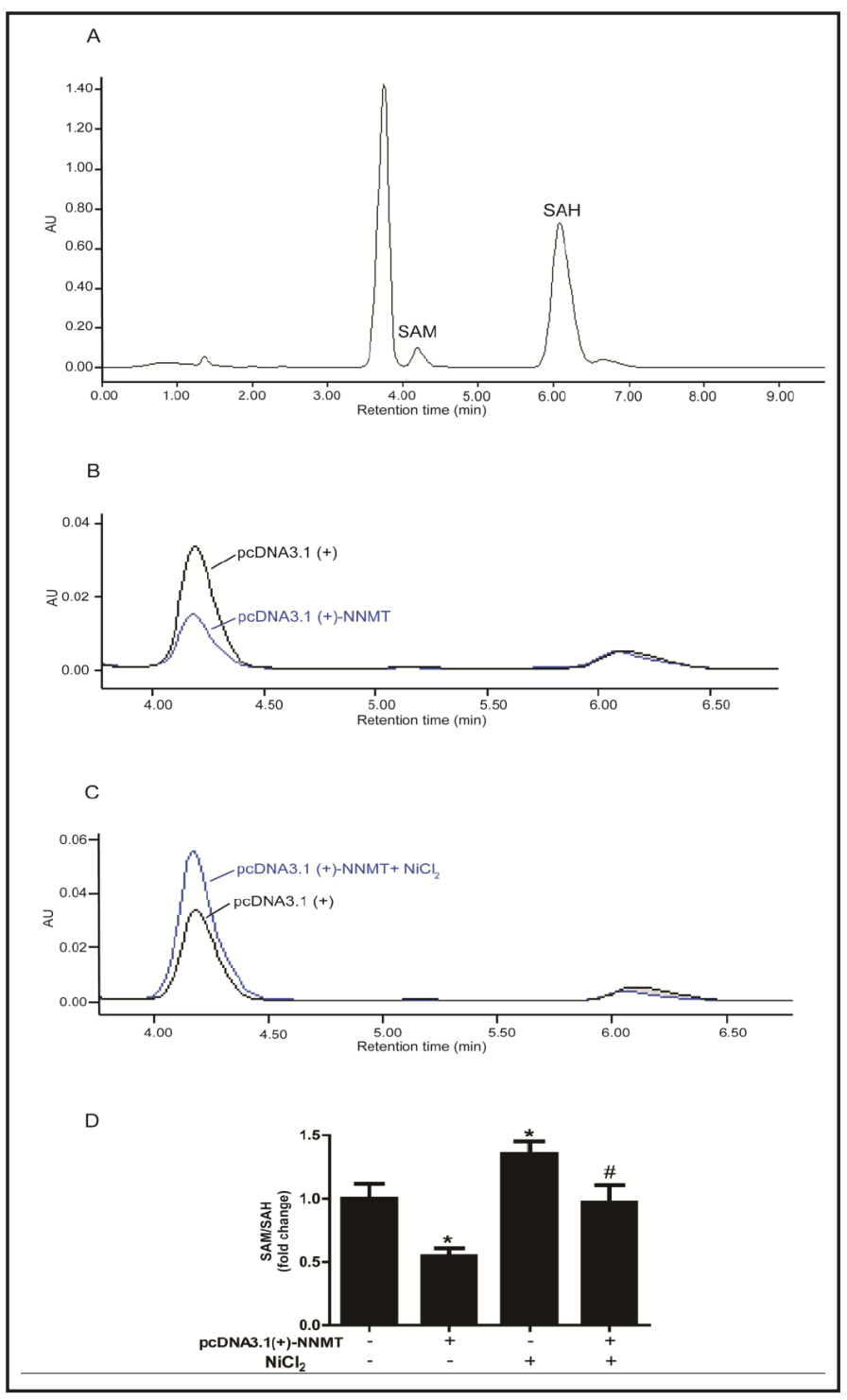

reduction in the SAM/SAH ratio, whereas nickel exposure led to a marked increase (Fig. 6B and C).

\section{Discussion}

In the present study, we found that H3K9 dimethylation was elevated after nickel exposure . In addition, we demonstrated that MAP2K3 and DKK1, two tumor suppressor genes in lung cancer, were down-regulated by nickel. A recent study showed that increased $\mathrm{H} 3 \mathrm{~K} 9 \mathrm{me} 2$ in the gene promoters of MAP2K3 and DKK1 was associated with suppression of their expressions [27]. Therefore, our present study indicated that elevation of H3K9me2 may silence these tumor suppressor genes after nickel exposure.

The homeostasis of histone methylation is maintained by the balance between methylation and demethylation, which are controlled by histone methyltransferases (HMTs) and histone demethylases. Previous studies have shown that nickel increased H3K9 dimethylation by inhibiting the activity of Fe(II)-2-oxoglutarate-dependent histone H3K9 demethylase accompanied by inhibition of G9a methyltransferase [11, 32]. Further studies showed that histone-modifying enzymes, including acetyltransferases and 
Fig. 7. Nickel decreases H3K9 acetylation (H3K9ac), and over-expression of NNMT inhibits the nickel-induced decrease in H3K9ac. BEAS-2B cells were exposed to various concentrations of $\mathrm{NiCl}_{2}$ for $72 \mathrm{~h}$ or $200 \mu \mathrm{M} \mathrm{NiCl}_{2}$ for $0 \mathrm{~h}$ to $72 \mathrm{~h}$. Western blot analysis showed that nickel decreased H3K9ac levels in a dose-dependent (A) and time-dependent manner (B). BEAS2B cells were transfected with the pcDNA3.1(+) vector or pcDNA3.1(+)-NNMT for $24 \mathrm{~h}$ and then exposed to $200 \mu \mathrm{M} \mathrm{NiCl}_{2}$ for another 24 h. The protein expression of H3K9ac (C) was determined by western blot analysis. The data are normalized to the corresponding $\beta$-actin level. All data are presented as the mean \pm SEM. ${ }^{*} \mathrm{P}<0.05$, compared with the control group; $\# \mathrm{p}<0.05$, compared with nickel exposure alone.

methyltransferases, consumed the cellular intermediate metabolites acetyl-CoA and SAM as acetyl and methyl donors to promote histone acetylation and methylation, respectively [33]. A reduction in intracellular acetyl-CoA concentrations decreases histone acetylation, indicating that histone acetyltransferases may be sensitive to changes in the acetyl-CoA/ CoA ratio [34]. NNMT, an enzyme that metabolizes SAM, has a role in adipose tissue energy metabolism and cancer aggressiveness resulting from alterations in histone methylation [19, 23]. In our present study, we found that nickel-induced NNMT suppression mediated H3K9 dimethylation, and NNMT over-expression could reverse the decrease in H3K9 acetylation (H3K9ac) caused by nickel in BEAS-2B cells (Fig. 7). These results indicated that NNMT may act as a key regulator in nickel-induced histone modifications.

Our study revealed that NNMT abolished H3K9 dimethylation by decreasing the SAM/ SAH ratio. However, this process may not represent a universal mechanism for regulation of histone methylation because both mono- and trimethylated H3K9 were not increased after nickel exposure. These observations were partially consistent with a report showing that NNMT failed to regulate all histone methylation events in cancer cells [19]. Our previous study investigating the impact of nickel exposure on cellular energy metabolism revealed that nickel inhibited the mitochondrial respiratory chain and consequently decreased the $\mathrm{NAD}^{+} / \mathrm{NADH}$ ratio [21]. The linkage between nickel-induced histone methylation and $\mathrm{NAD}^{+} /$ NADH was further demonstrated by the results in our present study. We found that the NADH oxidant PMS efficiently suppressed the increase in $\mathrm{H} 3 \mathrm{~K} 9 \mathrm{me} 2$ and the decrease in $\mathrm{NAD}^{+}$/ NADH triggered by nickel (Fig. 5). NNMT catalyzes N-methylation of NAM by transferring a methyl group from SAM to NAM and generating SAH and MNA, thus lowering the levels of SAM available for methylation reactions of histones [35, 36]. The observed reduction in H3K9me2 levels by PMS may be correlated with the enhancement of NNMT activity because elevation of $\mathrm{NAD}^{+} / \mathrm{NADH}$ was positively associated with the activity of NNMT [37]. Moreover, NAM is a precursor for NAD $\mathrm{Ni}^{+}$biosynthesis through a salvage pathway. Based on the report that NAM reduced H3K4 trimethylation [38], it is tempting to speculate that more cellular NAM 
Fig. 8. PMS treatment fails to reverse nickel-induced repression of NNMT. NNMT protein level (A) was determined by western blot analysis. mRNA level of NNMT (B) was detected by qPCR analysis. All data are presented as the mean \pm SEM from at least three independent experiments. $* \mathrm{p}<0.05$, compared with the control.

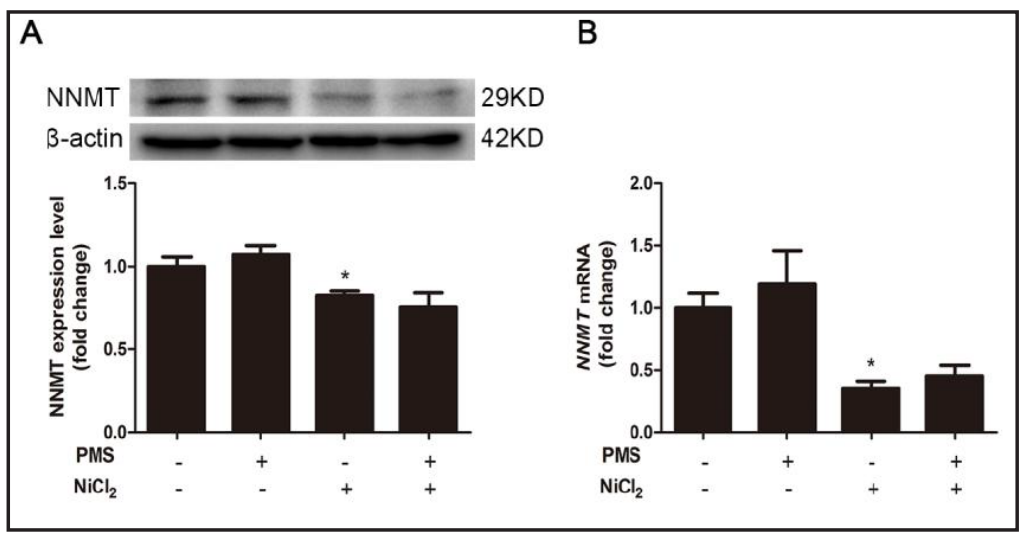

should be consumed in response to nickel-induced reduction of $\mathrm{NAD}^{+}$than that available for methylation catalyzed by NNMT, consequently inhibiting NNMT activity and leading to an increase in histone methylation. However, alterations in $\mathrm{NAD}^{+} / \mathrm{NADH}$ were not involved in nickel-induced NNMT repression at both the mRNA and protein levels (Fig. 8). Thus, the mechanism by which nickel suppressed NNMT expression remains to be elucidated in further studies.

In summary, our study is the first to report that nickel exposure suppresses NNMT in BEAS-2B cells. Over-expression of NNMT abolished the increase in nickel-induced H3K9me2 and resulted in a SAM/SAH ratio reduction. We propose that NNMT may be a key regulator of nickel-induced histone methylation by altering the cellular SAM/SAH ratio. These data revealed a new mechanism for nickel-induced histone methylation and linked the cross-talk between histone modifications and nickel toxicity.

\section{Acknowledgments}

This study was supported by grants from the National Natural Science Foundation of China (No. 81102108) and the Outstanding Youth Science Fund of Chongqing (No. CSTC2013JCYJJQ10002).

\section{Disclosure Statement}

The authors declare no conflicts of interest.

\section{References}

1 International Agency for Research on Cancer: IARC monographs on the evaluation of carcinogenic risks to humans, vol 49. Lyon, IARC, 1990.

- Ma L, Bai YN, Pu HQ, He J, Bassig BA, Dai M, Zhang YW, Zheng TZ, Cheng N: A retrospective cohort mortality study in Jinchang, the largest nickel production enterprise in China. Biomed Environ Sci 2014;27:567-71.

3 Kasprzak KS, Sunderman FW, Jr., Salnikow K: Nickel carcinogenesis. Mutat Res 2003;533:67-97.

4 Lu H, Shi X, Costa M, Huang C: Carcinogenic effect of nickel compounds. Mol Cell Biochem 2005;279:45-67.

5 Biggart NW and Costa M: Assessment of the uptake and mutagenicity of nickel chloride in salmonella tester strains. Mutat Res 1986;175:209-215.

6 Klein CB, Kargacin B, Su L, Cosentino S, Snow ET, Costa M: Metal mutagenesis in transgenic Chinese hamster cell lines. Environ Health Perspect 1994;102:63-67.

7 Arita A and Costa M: Epigenetics in metal carcinogenesis: Nickel, Arsenic, Chromium and Cadmium. Metallomics 2009;1:222-228. 


\section{Cellular Physiology Cell Physiol Biochem 2017;41:2016-2026

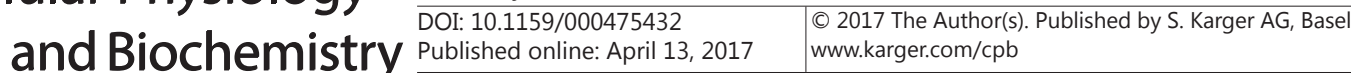 \\ Published onlring}

Li et al.: Nickel Induces H3K9me2 by Inhibiting NNMT

8 Dawson MA and Kouzarides T: Cancer epigenetics: from mechanism to therapy. Cell 2012;150:12-27.

$\checkmark 9$ Jenuwein T and Allis CD: Translating the histone code. Science 2001;293:1074-1080.

10 Peterson CL and Laniel MA: Histones and histone modifications. Curr Biol 2004;14:R546-51.

11 Chen H, Ke Q, Kluz T, Yan Y, Costa M: Nickel Ions Increase Histone H3 Lysine 9 Dimethylation and Induce Transgene Silencing. Mol Cell Biol 2006;26:3728-3737.

12 Jackson JP, Johnson L, Jasencakova Z, Zhang X, PerezBurgos L, Singh PB, Cheng X, Schubert I, Jenuwein T, Jacobsen SE: Dimethylation of histone H3 lysine 9 is a critical mark for DNA methylation and gene silencing in Arabidopsis thaliana. Chromosoma 2004;112:308-15.

-13 Chen H, Kluz T, Zhang R, Costa M: Hypoxia and nickel inhibit histone demethylase JMJD1A and repress Spry2 expression in human bronchial epithelial BEAS-2B cells. Carcinogenesis 2010;31:2136-2144.

-14 Scheller T, Orgacka H, Szumlanski CL, Weinshilboum RM: Mouse liver nicotinamide N-methyltransferase pharmacogenetics: biochemical properties and variation in activity among inbred strains. Pharmacogenetics 1996;6:43-53.

15 Yan L, Otterness DM, Craddock TL, Weinshilboum RM: Mouse liver nicotinamide N-methyltransferase: cDNA cloning, expression, and nucleotide sequence polymorphisms. Biochem Pharmacol 1997;54:11391149.

16 Yu T, Wang YT, Chen P, Li YH, Chen YX, Zeng H, Yu AM, Huang M, Bi HC: Effects of nicotinamide N-methyltransferase on PANC-1 cells proliferation, metastatic potential and survival under metabolic stress. Cell Physiol Biochem 2015;35:710-721.

-17 Bi HC, Pan YZ, Qiu JX, Krausz KW, Li F, Johnson CH, Jiang CT, Gonzalez FJ, Yu AM: N-methylnicotinamide and nicotinamide N-methyltransferase are associated with microRNA-1291-altered pancreatic carcinoma cell metabolome and suppressed tumorigenesis. Carcinogenesis 2014;35:2264-2272.

18 Pozzi V, Sartini D, Rocchetti R, Santarelli A, Rubini C, Morganti S, Giuliante R, Calabrese S, Di Ruscio G, Orlando F, Provinciali M, Saccucci F, Lo Muzio L, Emanuelli M: Identification and characterization of cancer stem cells from head and neck squamous cell carcinoma cell lines. Cell Physiol Biochem 2015;36:784-798.

19 Ulanovskaya OA, Zuhl AM, Cravatt BF: NNMT promotes epigenetic remodeling in cancer by creating a metabolic methylation sink. Nat Chem Biol 2013;9:300-306.

20 Chiarugi A, Dolle C, Felici R, Ziegler M: The NAD metabolome--a key determinant of cancer cell biology. Nat Rev Cancer 2012;12:741-752.

21 He MD, Xu SC, Zhang X, Wang Y, Xiong JC, Zhang X, Lu YH, Zhang L, Yu ZP, Zhou Z: Disturbance of aerobic metabolism accompanies neurobehavioral changes induced by nickel in mice. Neurotoxicology 2013;38:916.

22 Hong S, Moreno-Navarrete JM, Wei X, Kikukawa Y, Tzameli I, Prasad D, Lee Y, Asara JM, Fernandez-Real JM, Maratos-Flier E, Pissios P: Nicotinamide N-methyltransferase regulates hepatic nutrient metabolism through Sirt1 protein stabilization. Nat Med 2015;21:887-894.

23 Kraus D, Yang Q, Kong D, Banks AS, Zhang L, Rodgers JT, Pirinen E, Pulinilkunnil TC, Gong F, Wang YC, Cen Y, Sauve AA, Asara JM, Peroni OD, Monia BP, Bhanot S, Alhonen L, Puigserver P, Kahn BB: Nicotinamide $\mathrm{N}$-methyltransferase knockdown protects against diet-induced obesity. Nature 2014;508:258-262.

24 Ke Q Davidson T, Chen H, Kluz T, Costa M: Alterations of histone modifications and transgene silencing by nickel chloride. Carcinogenesis 2006;27:1481-1488.

-25 Ding J, He G, Gong W, Wen W, Sun W, Ning B, Huang S, Wu K, Huang C, Wu M, Xie W, Wang H: Effects of nickel on cyclin expression, cell cycle progression and cell proliferation in human pulmonary cells. Cancer Epidemiol Biomarkers Prev 2009;18:1720-1729.

26 Kim IG, Kim SY, Kim HA, Kim JY, Lee JH, Choi SI, Han JR, Kim KC, Cho EW: Disturbance of DKK1 level is partly involved in survival of lung cancer cells via regulation of ROMO1 and gamma-radiation sensitivity. Biochem Biophys Res Commun 2014;443:49-55.

27 Jose CC, Xu B, Jagannathan L, Trac C, Mallela RK, Hattori T, Lai D, Koide S, Schones DE, Cuddapah S: Epigenetic dysregulation by nickel through repressive chromatin domain disruption. Proc Natl Acad Sci U S A 2014;111:14631-14636.

-28 Park JC, Chae YK, Son CH, Kim MS, Lee J, Ostrow K, Sidransky D, Hoque MO, Moon C: Epigenetic silencing of human T (brachyury homologue) gene in non-small-cell lung cancer. Biochem Biophys Res Commun 2008;365:221-226.

29 Yamaki A and Muratsubaki H: Phenazine methosulfate decreases HIF-1alpha accumulation during the exposure of cells to hypoxia. Biosci Biotechnol Biochem 2012;76:1682-1687. 


\section{Cellular Physiology Cell Physiol Biochem 2017;41:2016-2026 and Biochemistry \begin{tabular}{c|c} 
DOI: 10.1159/000475432 \\
Published 2017
\end{tabular} \begin{tabular}{l}
$\begin{array}{l}\text { O 2017 The Author(s). Published by S. Karger AG, Basel } \\
\text { www.karger.com/cpb }\end{array}$ \\
\cline { 2 - 3 }
\end{tabular} \\ \begin{tabular}{lll} 
Published online: April 13, $2017 \quad$ www.karger.com/cpb \\
\hline
\end{tabular}}

-30 Fan J, Krautkramer KA, Feldman JL, Denu JM: Metabolic regulation of histone post-translational modifications. ACS Chem Biol 2015;10:95-108.

-31 Grothe J, Riethmuller J, Tschurtz SM, Raith M, Pynn CJ, Stoll D, Bernhard W: Plasma phosphatidylcholine alterations in cystic fibrosis patients: impaired metabolism and correlation with lung function and inflammation. Cell Physiol Biochem 2015;35:1437-1453.

-32 Chen H, Giri NC, Zhang R, Yamane K, Zhang Y, Maroney M, Costa M: Nickel ions inhibit histone demethylase JMJD1A and DNA repair enzyme ABH2 by replacing the ferrous iron in the catalytic centers. J Biol Chem 2010;285:7374-7383.

-33 Katada S, Imhof A, Sassone-Corsi P: Connecting threads: epigenetics and metabolism. Cell 2012;148:24-28

-34 Wellen KE, Hatzivassiliou G, Sachdeva UM, Bui TV, Cross JR, Thompson CB: ATP-citrate lyase links cellular metabolism to histone acetylation. Science 2009;324:1076-1080.

-35 Kang-Lee YA, McKee RW, Wright SM, Swendseid ME, Jenden DJ, Jope RS: Metabolic effects of nicotinamide administration in rats. J Nutr 1983;113:215-221.

-36 Varela-Rey M, Martinez-Lopez N, Fernandez-Ramos D, Embade N, Calvisi DF, Woodhoo A, Rodriguez J, Fraga MF, Julve J, Rodriguez-Millan E, Frades I, Torres L, Luka Z, Wagner C, Esteller M, Lu SC, MartinezChantar ML, Mato JM: Fatty liver and fibrosis in glycine N-methyltransferase knockout mice is prevented by nicotinamide. Hepatology 2010;52:105-114.

-37 Sternak M, Jakubowski A, Czarnowska E, Slominska EM, Smolenski RT, Szafarz M, Walczak M, Sitek B, Wojcik T, Jasztal A, Kaminski K, Chlopicki S: Differential involvement of IL-6 in the early and late phase of 1-methylnicotinamide (MNA) release in Concanavalin A-induced hepatitis. Int Immunopharmacol 2015;28:105-114.

-38 Xydous M, Sekeri-Pataryas KE, Prombona A, Sourlingas TG: Nicotinamide treatment reduces the levels of histone H3K4 trimethylation in the promoter of the mper1 circadian clock gene and blocks the ability of dexamethasone to induce the acute response. Biochim Biophys Acta 2012;1819:877-884. 